\title{
La construction identitaire chez les élèves d'un projet d'immersion réciproque italo-allemande à Francfort-sur-le-Main
}

\section{Melanie Kunkel}

\section{(2) OpenEdition \\ Journals \\ Édition électronique \\ URL : http://journals.openedition.org/trema/2578 \\ DOI : $10.4000 /$ trema. 2578 \\ ISSN : 2107-0997 \\ Éditeur \\ Faculté d'Éducation de l'université de Montpellier}

\section{Édition imprimée}

Date de publication : 1 décembre 2010

Pagination : 128 - 143

ISSN : 1167-315X

\section{Référence électronique}

Melanie Kunkel, « La construction identitaire chez les élèves d'un projet d'immersion réciproque italoallemande à Francfort-sur-le-Main », Tréma [En ligne], 33 - 34 | 2010, mis en ligne le 01 décembre 2012, consulté le 20 avril 2019. URL : http://journals.openedition.org/trema/2578; DOI : 10.4000/ trema. 2578

Ce document a été généré automatiquement le 20 avril 2019.

Trema 
La construction identitaire chez les élèves d'un projet d'immersion réciproque italo-allemande à Francfortsur-le-Main

Melanie Kunkel

I. Introduction : langue(s), école et identité 
1 La relation entre la construction identitaire et le plurilinguisme a été beaucoup discutée dans la littérature spécialisée; les conceptions modernes entendent l'identité comme un phénomène pluriel, complexe et changeant au cours de la vie (cf. HAARMANN 1996). Depuis quelques années, les travaux de VYGOTSKY, KRISTEVA et BOURDIEU sont mit en avant dans l'analyse du développement identitaire des individus bi- ou plurilingues suite aux processus de migrations. Le concept de l'identité ne vise pas seulement à caractériser une personne comme individu, mais aussi comme membre d'une communauté - linguistique, sociale, ethnique, etc. (cf. LE PAGE/TABOURETKELLER 1985 : 3) ; l'identité peut être liée à l'image qu'un individu se fait de lui-même et à l'image que les autres se font de lui. Si

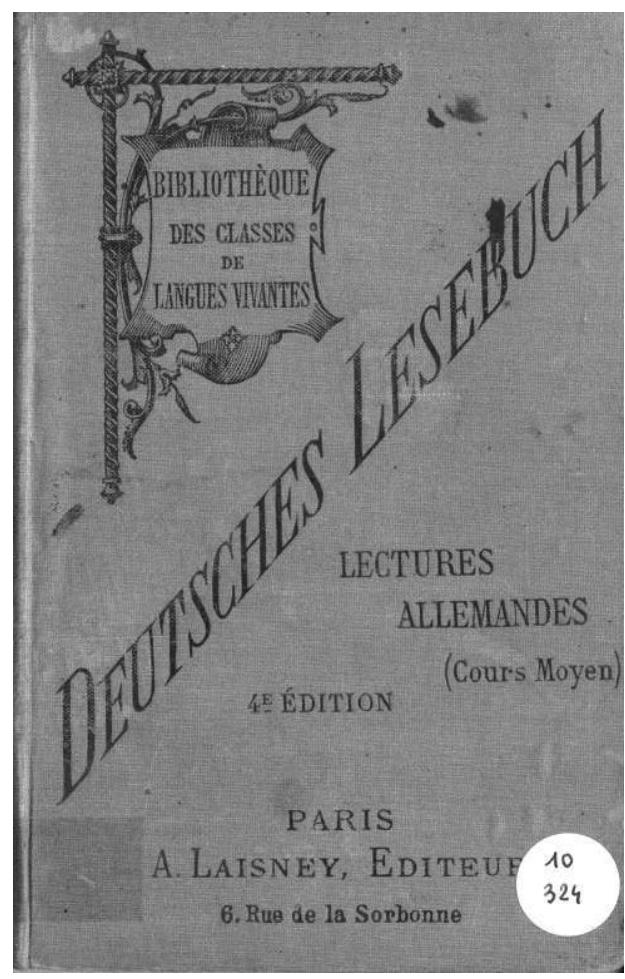
nous «identifions» un individu, notre jugement est basé sur des «signes»: l'apparence, la conduite, le comportement dans l'interaction sociale, mais c'est aussi la langue qui joue un rôle fondamental dans la définition identitaire d'un individu.

Même si dans les écoles un nombre toujours croissant d'enfants apporte une ou plusieurs langues d'origine outre la langue majoritaire, l'habitus monolingue (GOGOLIN 1994) détermine encore l'enseignement en France, en Allemagne et dans beaucoup d'autres pays officiellement monolingues. L'ouverture vers les langues d'origine des enfants outre la langue nationale ou d'autres langues de prestige valorisées par le curriculum officiel - est donc strictement limitée. Pour les enfants d'immigrés, cela peut avoir des conséquences fatales :

2 [T]his devaluation of identity played out in the interactions between educators and students convince many students that academic effort is futile. They resist further devaluation of their identities by mentally withdrawing from participation in the life of the school. [...] To turn this scenario around and reverse the pattern of academic failure inevitably requires that educators, students, and communities challenge the historical pattern of subordination that has characterized relations in the broader society. When educators encourage culturally diverse students to develop the language and culture they bring from home and build on their prior experiences, they, together with their students, challenge the perception in the broader society that these attributes are inferior or worthless. (CUMMINS $1996: 3)^{1}$

Dans la perspective de cette analyse, diverses approches d'éducation interculturelle cherchent à valoriser les ressources linguistiques et culturelles des élèves pour créer un climat de tolérance et d'estime réciproques ainsi que pour enrichir l'enseignement. Mais si l'on va au-delà, c'est le concept de l'immersion réciproque provenant des Etats-Unis, qui nous semble prédominer dans ces approches : au début de la scolarité, une classe est composée d'une moitié d'enfants partageant la même langue d'origine qui n'est pas la langue majoritaire (p. e. le turc, l'italien, le grec) et d'une autre moitié d'enfants qui ne 
savent pas (encore) cette langue. ${ }^{2}$ Les deux langues sont considérées comme des langues " partenaires » - cette désignation souligne l'équilibre souhaité.

L'immersion réciproque est jugée favorable à la valorisation des ressources linguistiques et culturelles des enfants d'immigrés et vise, par conséquent, à les soutenir dans le développement de leur personnalité, leur identité et leur confiance en eux. Cependant elle vise aussi à la communication interculturelle, à la capacité d'un changement de perspectives et de respect de l'autre. D'autres objectifs viennent complexifier ce phénomène: sur le plan linguistique, le projet vise la promotion d'un bilinguisme développé. Etant donné que les connaissances de départ linguistiques des élèves sont diverses, la collaboration entre les enfants, dès le début du parcours scolaire, est particulièrement renforcée pour les faire profiter mutuellement de leurs connaissances. Pour les enfants d'immigrés qui parlent la langue migratoire dans leur famille, il s'agit surtout de développer dans leur répertoire un registre académique formel auquel ils n'ont souvent pas accès chez eux. Mais l'échange entre les élèves n'est pas, bien entendu, limité au niveau linguistique ; il s'étend au champ culturel dans son ensemble. Un autre objectif important est de leur transmettre des connaissances suffisantes qui garantissent le succès scolaire. Parmi les enfants d'immigrés, ceux qui ne maitrisent pas suffisamment la langue majoritaire profitent d'un accès aux matières à travers leur L1.

Ainsi, l'immersion réciproque pourrait offrir une alternative aux conséquences négatives d'une assimilation linguistique forcée par l'école. Dans notre contribution, nous chercherons à approfondir l'aspect de la construction identitaire, la perspective du sujet plurilingue étant au centre de notre réflexion (cf. DE FLORIO-HANSEN \& HU [éds.] 2003). En analysant les points de vue d'un certain nombre d'élèves et en y appliquant la théorie de l'investissement (NORTON 1993, 2000), nous illustrerons l'impact qu'un tel projet peut avoir sur la construction et l'affirmation des identités individuelles et collectives des élèves.

\section{Le champ de recherche : projets bilingues d'immersion réciproque}

4 Les projets d'immersion réciproque dont le concept sous-jacent a été esquissé ci-dessus, peuvent, dans leur réalisation, différer selon plusieurs variantes : par exemple, la langue minoritaire choisie, la proportion des élèves appartenant à chaque groupe linguistique, la répartition du temps de l'enseignement sur les deux langues, la séparation (ou intégration) des langues à travers les matières ou les jours (LINDHOLM-LEARY 2001, 30-33). Pour illustrer le contexte de notre recherche, nous donnerons un aperçu de la réalisation du projet de Francfort.

Aux Etats-Unis, pendant l'année scolaire 2008/09, il existe 335 projets bilingues classifiés comme immersion réciproque selon les critères du Center for Applied Linguistics, Washington, avec plus de $90 \%$ des projets incluant l'espagnol comme langue partenaire à côté de l'anglais. ${ }^{3}$ L'Allemagne a suivi cet exemple en introduisant le modèle de l'immersion réciproque. Celui-ci a connu ses débuts dans les années 90 , quand la ville de Berlin a commencé d'établir peu à peu un réseau d'écoles publiques européennes (Staatliche Europaschulen Berlin) qui offrent - aujourd'hui - neuf différentes « langues partenaires » : l'anglais, l'espagnol, le français, le grec, l'italien, le polonais, le portugais, le russe et le turc (cf. GÖHLICH [éd.] 1998). Parmi les premiers projets se trouve aussi l'école italo-allemande (Deutsch-Italienische Gesamtschule) de Wolfsburg, ville marquée 
par l'industrie de Volkswagen et ensuite par l'immigration des travailleurs italiens dans les années 50-60 (cf. SANDFUCHS \& ZUMASCH 2002). L'italien est parmi les langues les plus représentées aussi dans d'autres projets d'immersion réciproque qui se trouvent par exemple à Hambourg, à Francfort-sur-le-Main, à Stuttgart et à Cologne (cf. HEINE \& RICCO \& SCHOOF-WETZIG [éds.] 2003).

Nous avons mené nos recherches à Francfort-sur-le-Main où, à la suite d'une initiative des familles italiennes ou binationales (cf. FILLIA 2003), en 1997 une section italoallemande a été ouverte dans une école élémentaire. Elle a été poursuivie dans un lycée, et entre-temps, la demande dépassant largement le nombre de places disponibles, il y a une deuxième école élémentaire qui possède une section bilingue. Les élèves de la classe bilingue, qui normalement est une classe sur trois ou quatre, doivent déjà avoir de bonnes connaissances d'italien au début du lycée, même s'ils n'ont pas suivi la classe bilingue au niveau élémentaire. Au lycée, environ deux tiers des élèves sont d'origine italienne ou mixte (italo-allemande, italo-roumaine, etc.), cependant la majorité d'entre eux représente la deuxième ou même la troisième génération d'immigrés. Une partie des cours est assurée par des enseignants italiens, délégués et payés par le ministère des affaires étrangères italien, qui de cette manière soutient le projet. Les matières telles que biologie, art, histoire et géographie ainsi que - selon les disponibilités des enseignants italiens - chimie et physique, sont enseignées dès le début soit en allemand soit en italien, en alternance selon les années scolaires. Une spécificité du projet est le team teaching: dans une partie des leçons de mathématiques et d'allemand/d'italien, un enseignant de langue allemande et un enseignant de langue italienne donnent le cours ensemble en alternant les langues, de sorte que l'échange interculturel ne commence pas au niveau des élèves (cf. KUNKEL \& BUDACH 2008; KUNKEL à par.), mais d'abord entre les enseignants (cf. KUNKEL 2008 ; BUDACH \& DREHER \& SPANU 2008). Le curriculum, outre les contenus officiellement prévus par le Land de Hesse, considère aussi des contenus du curriculum correspondant italien. Un programme intégré est développé au cours des années. Toutefois, l'allemand reste toujours dominant aux différents niveaux - quant au nombre des matières enseignées dans cette langue, à la communication entre les enseignants italiens et allemands, à l'administration de l'école, aux livres disponibles dans la bibliothèque, etc.

\section{Cadre théorique et études préliminaires}

5 Malgré l'objectif complexe des projets d'immersion réciproque esquissé ci-dessus, les travaux portant sur l'output linguistique et académique prévalent encore longtemps sur les travaux qui insistent sur les buts plus difficiles à cerner, comme l'apprentissage interculturel ou la construction identitaire. Les résultats des études sur la performance linguistique et académique des élèves démontrent en général un effet positif des projets (cf. THOMAS \& COLLIER 1997, 2002 ; LINDHOLM-LEARY 2001) ${ }^{4}$.

Des recherches ethnographiques ont récemment mis l'accent sur des problèmes de statut de langue et d'identité ethnique. Par exemple, AMREIN \& PEÑA (2000) démontrent qu'un vrai équilibre entre les langues, à tous les niveaux, demeure encore une vision inaccessible - même dans les projets d'immersion réciproque. MCCOLLUM (1999) décrit une prédominance de la langue majoritaire dans un projet anglais-espagnol qu'elle attribue à une dévalorisation des variétés de l'espagnol apportées par les élèves et à un plus grand prestige de la langue anglaise. En interprétant les résultats d'une enquête 
menée parmi les élèves d'un projet d'immersion réciproque, BEARSE \& DE JONG (2008) constatent des différences dans les investissements identitaires entre les étudiants anglophones et hispanophones ; l'espagnol reste une langue d'école pour les anglophones, la motivation étant instrumentale, tandis que pour les élèves latino-américains, la langue espagnole fait partie de leur identité, étant par essence la langue de la famille et non celle de l'école. D'après l'étude de POTOWSKI $(2005,2007)$, qui a analysé la construction d'identités des élèves à travers les pratiques langagières dans les branches d'immersion réciproque, l'investissement dans la langue et la culture d'origine est dominé par celui dans la langue et la culture majoritaires.

Le concept de l'investissement, dont BEARSE \& DE JONG et POTOWSKI se sont servies, nous semble un instrument d'analyse approprié pour aborder notre problématique portant sur les représentations de l'individu plurilingue. Norton Peirce (1995) a introduit le concept d'investissement pour désigner l'attitude des apprenants à l'égard de la langue cible, qui se construit dans un contexte social et historique. L'auteur a recours à des notions économiques utilisées par Bourdieu : les apprenants investissent dans une langue lorsqu'ils espèrent en acquérir des ressources symboliques et matérielles, un "return » qui justifie leur investissement. En se distinguant des concepts de motivation (cf. GARDNER \& LAMBERT 1972), elle souligne l'importance du contexte pour l'apprentissage d'une langue :

6 [...] I take the position that notions of the individual and the language learner's personality in SLA theory need to be re-conceptualized in ways that will problematize dichotomous distinctions between the language learner and the language learning context. I use the term identity to reference how a person understands his or her relationship to the world, how that relationship is constructed across time and space, and how the person understands possibilities for the future. I argue that SLA theory needs to develop a conception of identity that is understood with reference to larger, and frequently inequitable, social structures which are reproduced in day-to-day social interaction. (Norton $2000: 5)^{5}$

7 En tenant compte de l'environnement social et familial des élèves, comme il ressort des interviews menés avec trois filles, et surtout du concept et de la réalisation du projet bilingue dans lequel elles apprennent les langues, nous chercherons à décrire et à analyser leur investissement.

\section{Méthodologie et recueil des données}

Nous nous référons à une méthodologie de recherche interprétative - ethnographique déjà bien établie dans la sociolinguistique qui s'étend depuis un temps récent dans la didactique des langues étrangères (cf. HU 2001, 2003). En conformité avec le concept de l'investissement établi par Norton Pierce, dans cette méthodologie, l'apprentissage et l'enseignement des langues sont considérés non seulement comme un processus individuel, mais comme une action sociale et culturelle à l'intérieur d'institutions, encadrée par un contexte socioculturel. La recherche ethnographique cherche à saisir une situation en tenant compte de toute sa complexité et en s'intéressant aux points de vue des acteurs qui ressortent des interviews et des observations. En outre, le processus de recherche est accompagné d'une réflexion critique du rôle du chercheur.

Notre recherche, fondée sur les observations participantes (LÜDERS 1995) en salle de classe et sur des entretiens semi - narratifs d'une durée moyenne de 90 à 120 minutes menés avec des élèves de 14 à 18 ans, ${ }^{6}$ a pour but principal d'analyser la perception 
générale que les élèves ont du bilinguisme et du projet bilingue. Les sujets de la biographie langagière, de l'usage linguistique et des expériences acquises dans le projet bilingue constituent le fil conducteur de l'interview.

Dans le cadre de cette contribution nous insistons sur la question de la construction identitaire des élèves et nous présentons puis interprétons quelques données relatives à ce sujet. En particulier, nous faisons référence aux explications des élèves qui considèrent leur biographie langagière (comment ta vie jusqu'ici s'est-elle déroulée du point de vue des langues?) et à l'image qu'ils se font d'eux-mêmes (comment te présenterais-tu à quelqu'un d'autre?).

\section{V. Étude de cas}

9 Par la suite, pour donner une impression des constructions identitaires, nous présenterons des esquisses de trois élèves fréquentant deux classes différentes du projet bilingue : une classe de troisième et une de seconde. Si nous choisissons une élève avec deux parents allemands, une autre avec une mère italienne et un père allemand et une dernière avec deux parents d'origine italienne, dans la conception d'une approche ethnographique, cela ne peut pas avoir, bien sûr, une prétention de représentativité ${ }^{7}$ : la recherche ethnographique ne vise pas une généralisation des résultats, mais une meilleure compréhension de situations complexes.

Pendant la phase d'observation participante, qui a précédé les interviews, nous nous sommes concentrée sur l'usage linguistique en classe. Tandis qu'avec les enseignants italiens et pendant les discussions dirigées par ceux-ci, les élèves maintiennent un discours en italien, ils utilisent entre eux presque exclusivement l'allemand - un résultat qui correspond à celui des recherches menées aux Etats-Unis (cf. supra). Quelques exceptions, rares, concernent surtout les situations où les élèves ont besoin d'une « langue secrète "; quand, dans d'autres occasions, ils font des plaisanteries ou des jeux de mots en italien, ce sont surtout les élèves italiens qui y participent.

\section{1. Luise : l'ouverture vers une autre identité}

10 Luise a 15 ans ; elle appartient à la minorité des élèves provenant de familles allemandes sans aucun parent italien ou d'origine autre qu'allemande. En dehors des communications menées avec l'enseignant italien ou contrôlées par lui, Luise, comme les autres élèves de la classe, n'utilise presque jamais l'italien à l'école. Les situations où l'italien lui sert de «langue secrète » avec son frère - qui lui aussi a fréquenté le projet bilingue pendant quelques années - et avec ses camarades de classe restent une exception. Elle souligne que ce sont surtout les séjours à l'étranger comme l'échange scolaire avec une classe de quatrième à Milan qui l'ont, en lui enlevant la peur de parler, fait avancer dans la capacité de s'exprimer en italien. Depuis l'année dernière, Luise a aussi fait des voyages en Italie avec sa copine Chiara, parlant l'italien avec la famille italienne ou d'autres jeunes. Elle aime aussi mettre à l'épreuve ses connaissances de l'italien pendant les vacances avec sa propre famille. Depuis quelque temps elle cherche à parler de temps en temps l'italien en classe avec ses camarades, suivant le conseil de ses enseignants, mais elle - comme les autres - considère comme difficile le fait de maintenir cette langue pendant toute la conversation. 
[...] dass man eigentlich auch besonders in diese Kultur reinkommt, also dass man einfach wie gesagt auch dann da drinne ist. Man wird sehr verbunden, man wird auch nicht irgendwie ausgegrenzt als deutsch oder italienisch und so, sondern wir sind EINE klasse [...]. Wir sind wie, wie so ein zusammengewürfelter Haufen, der aber doch trotzdem irgendwie die italienische oder die italienisch-deutsche Kultur nicht miteinander vergleicht, aber doch manchmal guckt, wie das denn unterschiedich ist, und wir auf jeden Fall mehr, vor allem für mich jetzt, mehr über die Kultur auch kennenlernen.
[...] que I'on entre dans la culture, que Ion est dedans, comme j'ai déjà dit. On s'attache, on n'est pas exclus en tant qu'allemand ou italien ou autre, mais or est UNE SEULE classe [...]. On est comme une foule de personnes mélangées, qui, par contre, ne compare pas la culture italienne, la culture italo-allemande l'une avec l'autre, mais qui, plutôt, cherche à comprendre ce qui diffère lune de l'autre, et on apprend beau. coup plus - et, moi personnellement, fapprends beaucoup plus sur la culture.

11 Ici, Luise confirme l'idée d'un échange culturel entre les élèves; elle semble avoir intériorisé les stratégies de coopération promues par ce projet (cf. supra). Luise se sent « dedans » l'autre culture et elle souligne plusieurs fois l'unité de la classe (« on est UNE SEULE classe »): les élèves ne sont pas répartis d'après leur nationalité ou leurs connaissances linguistiques, mais ils étudient tous ensemble les cultures italienne et allemande. De temps en temps, il y a la possibilité de découvrir des différences entre les deux cultures. Luise souligne que pour elle, c'est un enrichissement d'apprendre quelque chose sur la culture italienne - notamment grâce aux connaissances de ses copains de classe. Cette perception qu'elle a gagnée de cette étroite collaboration la mène à une expérience personnelle qu'elle décrit comme suit.

\section{Extrait 2 : Luise}

Ich würd mich jetzt auch nicht mehr als typisch deutsch bezeichnen, weil ich das mitgemacht hab, sondern ich würd selber sagen, dass ich eigentlich ein Stück weit, vielleicht jetzt nicht italienisch aut dem Pass oder so, aber dass ich mit der Sprache so weit konfrontiert bin, dass ich mich auf jeden Fall so ein bisschen dazuzählen kann, weil ich eigentlich auch die Lebenseinstellung [...], man hat sich dadurch ein bisschen verändert und eine andere Perspektive bekommen.
Ayant participé à ce projet, je ne me considere plus comme une allemande typique, mais je dirais que je suis un petit peu - pas italienne sur le passeport ou autre, mais que je suis tellement confrontée avec la langue, parce que je peux m'y intégrer un petit peu, comme je partage leur conception de la vie [...], on a un peu changé et on a adopté une autre perspective.

Ici, Luise laisse apercevoir certains impacts sur son identité : son expérience dans le projet bilingue la pousse jusqu'à dire qu'elle ne se voit plus comme « allemande typique ». En distinguant entre sa nationalité "sur le passeport», qui, bien entendu, est restée allemande, et une "conception de la vie ", une "autre perspective " qu'elle a gagnée grâce au projet, elle se trouve enrichie. La confrontation intensive avec la langue italienne, pour elle, y joue un rôle fondamental ( «je suis tellement confrontée avec la langue »). A la fin, en passant de la première à la troisième personne («on a un peu changé »), elle semble généraliser cet effet au niveau de la classe bilingue. Pourtant, elle reste très mesurée dans la formulation de ce changement (« un peu ») de manière telle 
qu'elle y attribue plutôt le statut d'une ouverture que de l'acquisition d'une véritable deuxième identité.

\section{2. Chiara : « deuxième langue maternelle » ou « première langue étrangère »?}

Chiara a 16 ans ; du côté paternel, sa famille est allemande ; seule la grand-mère du côté maternel est italienne, mais la mère, comme Chiara, est née à Francfort. Pendant son enfance, Chiara n'a parlé que l'allemand avec sa famille. Elle dit se souvenir qu'elle regrettait ne pas comprendre ce que sa mère disait en italien à la grand-mère ou à d'autres parents. Avec la scolarisation, elle commence à apprendre l'italien; mais même aujourd'hui, elle ne parle l'italien avec sa mère et sa grand-mère que rarement - surtout dans les cas où la communication devient en quelque sorte "émotionnelle ». Sa mère apprécie beaucoup les connaissances linguistiques de sa fille qu'elle-même n'a pas réussi à lui transmettre; et lors des visites en Italie, Chiara a maintenant l'occasion de parler l'italien avec sa famille. Avec ses amis à Francfort, elle parle presque toujours l'allemand; seulement lors des activités dans la paroisse catholique italienne elle déclare parler l'italien. Au cours de l'interview, Chiara hésite entre désigner l'italien comme "sa deuxième langue maternelle » et "sa première langue étrangère ». Un tel conflit apparaît aussi dans d'autres passages de notre interview. Ainsi, en réfléchissant aux débuts du projet, Chiara nous raconte des problèmes de sa catégorisation comme « italienne ».

Chiara

\begin{abstract}
Also am Arfang oder, ich sag mal, so bis zur sechsten Klasse oder so war es so, dass wir auch immer drauf Rücksicht genommen haben, wer hat jetzt italienische Familie, wer ist komplett italienisch oder wer ist deutsch. Also, da haben sie auch bei den Benotungen immer Unterschiede gemacht und haben gesagt: $O$. K., dafür dass die Person deutsch ist und wirklich NICHTS kann, ist das dann 'ne Zwei, und der andere hat dann halt auch ne Zwei bekommen, also der Italiener [...]. Und das hat mich dann früher halt immer total aufgeregt, weil ich wurde immer als italienisch eingestuft sozusagen, obwoht ich überhaupt nicht WAA, und ich hab auch zu Hause NIE italienisch geredet, also früher nicht, und deswegen, dann hatten wir dann auch ofters mal Probleme und haben mit den Lehrem geredet [...]. Aber es war doch. irgendwo hat es schon Sinn, also das fand ich, ja.
\end{abstract}

\begin{abstract}
Au début, je dirais jusqu'à la sixième environ, on a pris en consideration quel élève a une famille italienne, qui est complètement italien ou qui est allemand. Alors, là, ils ont tait des différences en attribuant des notes, et lis ont dit : o. k., vu que la personne est allemande et ne sait RIEN, c'est ,mention bien", et l'autre a aussi obtenu ,mention bien", Iitalien [...]. Et ça m'a toujours enervée parce que moi, j'étais classée comme une italienne, même si je NE L'ETAIS PAS, et je n'ai JAMAIS parlé litalien chez moi au début. Et ça a souvent provoqué des problèmes et on en a parlé avec les enseignants [...]. Mais d'une certaine manière, ca a du sens, je trouve.
\end{abstract}

Dans ses souvenirs, la répartition conceptuelle des élèves selon leur origine («complètement italien », «italien - allemand» et "allemand») qui a pour but de prendre en compte leurs bases linguistiques de départ en attribuant les notes, mène à des conflits. Même si Chiara, qui porte un prénom italien et un nom allemand, a une mère italienne, elle souligne qu'elle "n'était pas italienne ", ce qui pour elle est étroitement lié avec le fait qu'elle n'avait jamais parlé l'italien chez elle. Pour expliquer la situation, 
Chiara et sa mère cherchent le dialogue avec les enseignants. Mais l'exemple confirme aussi que les ressources linguistiques des élèves italiens dans ce projet sont reconnues : au début de la scolarisation, les critères appliqués sont adaptés au niveau d'entrée des élèves de manière que les élèves d'origine italienne sachent qu'ils sont jugés plus compétents dès le départ. Leur rôle d'experts parmi les élèves est donc officialisé. Quand Chiara confirme que cette approche «a du sens», elle souligne accepter cette idée constitutive.

\section{3. Valentina : le rôle du standard pour l'ouverture d'autres espaces communicatifs} Allemagne; ses grands-parents vivent en Allemagne aussi. La famille parlait le sicilien avec Valentina, quand elle était petite ; aujourd'hui, la conversation se déroule presque exclusivement en allemand avec les parents, tandis qu'elle parle en sicilien ou en italien avec ses grands-parents. Pendant les vacances, elle séjourne régulièrement chez la famille en Italie. Même si elle est convaincue de mieux parler l'allemand, elle se sent " plus à l'aise » en italien. Valentina souligne son identité italienne.

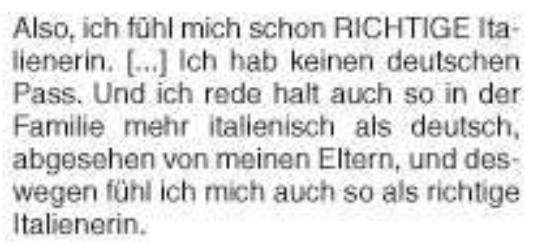

Alors, je me sens une vraie Italienne. [...] Je n'ai pas de passeport allemand. Et dans la famille, à part mes parents, je parle aussi plus ditalien que d'allemand, et c'est pourquoi je me sens comme une vraie Italienne.

Pour elle, une identité italienne est liée d'un côté au passeport, de l'autre à l'italien comme langue de famille. Pour mieux illustrer son concept d'identité, elle nous raconte, après le passage cité, l'histoire d'un ami turc qui ne parle pas le turc et qui, en conséquence, se voit comme " allemand ». Valentina, pendant l'interview, réfléchit aussi à la relation entre le sicilien et l'italien. Elle aime bien le dialecte, mais elle donne aussi une grande importance à l'apprentissage de la langue standard et à l'enrichissement du vocabulaire, car, pour faire ses études, Valentina projette d'aller en Italie après l'école. C'est avec fierté qu'elle raconte que ses parents lui demandent quelques fois des paroles en italien standard qu'eux-mêmes, n'ayant pas fréquenté une école italienne ou bilingue, ne connaissent pas. Valentina attribue au projet bilingue la possibilité de pouvoir participer à la vie publique en italien. 
Ich rede auch so nicht nur zu Hause, sondern auch in der Offentlichkeit aut Italienisch, hab damit hait kein Problem mehr. Früher hab ich mich so zurückgezogen, wollt nie italienisch reden aus Angst, dass ich Fehler mache. [...] Wobei halt ich hab das RICHTIGE Italienisch durch die Schule gelernt, sonst hab ich ja nur Dialekt gesprochen. Also es ist dank der Schule eigentlich so gewesen, dass ich die RICHTIGE Sprache kennengelernt habe.
Je ne parle seulement chez moi, mais aussi en public, là je n'ai plus de problème. Autrefois, je restais en retrait, je ne voulais jamais parler litalien par peur de faire des fautes. [...] J'ai appris ritalien CORRECT par l'école, en dehors ie ne parlais que le dialecte. C'est donc grâce à lécole que jaa appris à connaître la langue $\mathrm{COA}$ RECTE.

17 Elle raconte avoir surmonté la peur d'utiliser l'italien hors du cadre familial («autrefois, je restais en retrait »), avoir donc une meilleure confiance dans ses connaissances linguistiques.

18 Le projet bilingue de l'école lui a donc permis d'apprendre la langue standard en utilisant ses connaissances dialectales comme base de départ, et de cette manière l'a aidée à exploiter d'autres espaces communicatifs. ${ }^{9}$

\section{Conclusion}

19 A travers ces extraits nous constatons que les trois élèves reconnaissent les ressources plurilingues et pluriculturelles de leurs répertoires construites à l'intérieur et à l'extérieur de l'école. Selon ces représentations, telles qu'elles apparaissent à travers les extraits, nous pouvons ainsi induire que, dans l'apprentissage des langues, elles s'investissent de manière différente et avec des objectifs différents.

Quant aux ressources symboliques et matérielles qui sont au centre de la théorie de l'investissement de Bonny Norton, Luise souligne élargir son horizon personnel, s'intégrer dans la communauté de sa classe bilingue et gagner outre la connaissance d'une autre langue une autre "perspective». Pour Chiara, par contre, il est important de maintenir un lien avec sa famille en Italie, de construire et de maintenir sa propre identité comme italophone. Valentina, qui parle le dialecte depuis son enfance, attribue au projet la maîtrise du standard italien qui lui permet de "parler en public » et de pouvoir projeter de faire ses études en Italie. Et la construction identitaire? D’abord, il est remarquable que les élèves, elles-mêmes, voient toutes leur identité comme étroitement liée avec les langues parlées. Tandis que Luise nous parle directement d'une ouverture en direction d'une seconde identité gagnée grâce à la confrontation intensive avec l'italien, ${ }^{10}$ dans le discours de Chiara, à travers sa désignation de la langue italienne, on note des marques d'hésitation entre deux identités. Valentina souligne l'importance de l'usage de la langue italienne pour maintenir son identité italienne. L'analyse de ces cas s'allie à l'hypothèse susmentionnée que l'identité d'élèves en minorité peut être affirmée dans un projet bilingue, qui crée un contexte favorable à leur investissement dans la langue et culture italienne. Mais en plus, comme nous l'avons démontré dans le cas de Luise, elle peut même mener à ce que les autres élèves perçoivent une ouverture de leur propre identité vers une autre.

Tandis que les trois élèves ont décidé de suivre le projet bilingue et d'y investir le travail nécessaire pour les motifs qu'elles savent nommer explicitement, en même temps, leurs 
propres analyses confirment nos observations en salle de classe : la langue non marquée des interactions sociales parmi les élèves est l'allemand. Comment expliquer ce qui apparait comme une contradiction?

La description de la mise en oeuvre du projet à l'école (cf. supra) suggère une dominance de l'allemand à l'école sous beaucoup d'aspects. Mais aussi hors de l'école, l'allemand, pour la plupart des jeunes, est la langue dominante. Il n'est donc pas surprenant que cette dominance influe sur l'usage linguistique à l'école, comme les études ci mentionnées le confirment aussi pour les projets aux Etats-Unis: FREEMAN (1998: 185) parle de « leakage » (infiltration) de l'usage de la langue de la majorité dans la salle de classe. Les efforts pour changer cette situation de fond ne nous semblent pas prometteurs, surtout dans les classes avec un fort pourcentage d'élèves de la troisième génération d'immigrés ou de couples mixtes. Par contre, comme le suggère POTOWSKI (2004: 96), il est d'autant plus nécessaire d'encourager les élèves à utiliser la langue italienne pendant des cours, en mettant à leur disposition les moyens de communication nécessaires.

Pourtant, nous soulignerons qu'il ne faut pas perdre de vue que les trois jeunes profitent d'autres occasions pour parler l'italien en dehors de la salle de classe: pour Chiara et Valentina, c'est surtout lors des visites à leurs familles en Italie; mais Luise elle aussi cherche à parler l'italien avec les familles italiennes de ses copines ou pendant les vacances qu'elle passe en Italie ou avec sa famille ou avec ses copines chez leurs familles italiennes. Ici, le projet bilingue ouvre également des occasions de contact qui sont explicitement souhaitées (cf. HALLER \& PAGLIUCA ROMANO 2002, 9).

Il est nécessaire aussi de revenir sur la fonction de l'italien comme "langue secrète ", dans laquelle nous reconnaissons une contribution à la construction d'une identité comme "classe bilingue »: l'intégration des membres à l'intérieur du groupe et la délimitation face à l'extérieur. Il est à souligner que dans cette fonction tous les membres du groupe sont inclus, même ceux qui normalement ne participent pas aux entretiens en italien, comme Luise. ${ }^{11}$

Notre analyse a accentué la complexité de la construction identitaire de trois élèves d'origine différente dans un projet d'immersion réciproque et a donné un aperçu de l'effet positif qu'un tel projet peut avoir sur l'investissement identitaire - et donc pour l'apprentissage d'une langue. Nous considérons d'une grande importance de prendre en compte cette connexité inévitable entre identité et apprentissage d'une langue pour pouvoir mieux évaluer les effets de divers modèles d'éducation bilingue.

\section{BIBLIOGRAPHIE}

AMREIN, A., PEÑAROBERT, A. (2000), « Asymmetry in dual-language practice: Assessing imbalance in a program promoting equality ", in: Education Policy Analysis Archives 8 (8) (http://epaa.asu.edu/ epaa/v8n8.html, 22.8.2009).

BEARSE, C., DE JONG ESTER, J. (2008), « Cultural and Linguistic Investment: Adolescents in a Secondary Two-Way Immersion Program », in: Equity \& Excellence in Education 41 (3), pp. 325-340.

BUDACH, G., DREHER, U., SPANÙ, P. (2008), « Se non è chiaro prendete l'abaco.' Wege zu einem bilingualen Curriculum im Schnittpunkt von Multimodalität und Tandem-Lehren », in: BUDACH Gabriele, ERFURT Jürgen, KUNKEL Melanie (éds.) (2008), Écoles plurilingues - multilingual schools: Konzepte, Institutionen und Akteure, Frankfurt/M. et al., Peter Lang, pp. 291-316. 
CUMmINS, J. (1996), Negotiating identities: Education for empowerment in a diverse society, Los Angeles, California Association for Bilingual Education.

DE FLORIO-HANSEN, I., HU A. (ÉDS.) (2003), Plurilingualität und Identität. Zur Selbst- und Fremdwahrnehmung mehrsprachiger Menschen, Tübingen, Stauffenburg.

FILLIA, E. (2003), « Das bilinguale deutsch-italienische Schulprojekt in Frankfurt am Main », in: ERFURT Jürgen, BUDACH Gabriele, HOFMANN Sabine (éds.) (2003), Mehrsprachigkeit und Migration. Ressourcen sozialer Identifikation, FRANKFURT M. et al., Peter LANG, pp. 221-226.

FREEMAN REBECCA, D. (1998), Bilingual education and social change, Clevedon, UK, Multilingual Matters.

GARDNER, R. C., LAMBERT, W. E. (1972), Attitudes and Motivations in Second Language Learning, Rowley, Newbury House Publishers.

GOGOLIN, I. (1994), Der monolinguale Habitus der multilingualen Schule, Münster, New York, Waxmann.

GÖHLICH, M. (ÉD.) (1998), Europaschule - Das Berliner Modell. Beiträge zu zweisprachigem Unterricht, Europäischer Dimension, Interkultureller Pädagogik und Schulentwicklung, Neuwied, Kriftel, Luchterhand.

HAARMANN, H. (1996), « Identität », in: GOEBL Hans, NELDE PETER H., STARÝ Zdenek, Wölck Wolfgang (éds.), Kontaktlinguistik: Ein internationales Handbuch zeitgenössischer Forschung, Berlin, de Gruyter, pp. 218-233.

HALLER, I., PAGLIUCA, R. A. (2002), Auf der Reise nach Europa. In viaggio verso l'Europa. Bilinguales Lernen an einer Grundschule, Witten, pad.

HEINE, M., RICCÒ, A., SCHOOF-WETZIG, D. (ÉDS.) (2003), Bilinguales Lernen im interkulturellen Kontext, Braunschweig, Westermann.

HU, A. (2001), « Zwischen Subjektivität und dem Anspruch auf Exploration authentischer Perspektiven: Forschungsmethodische Anmerkungen zu einer interpretativ-ethnografischen Studie », in: MÜLLER-HARTMANN Andreas, SCHOCKER-VON DITFURTH Marita (éds.) (2001), Qualitative Forschung im Bereich Fremdsprachen lehren und lernen, Tübingen, Narr, pp. 11-39.

HU, A. (2003), Schulischer Fremdsprachenunterricht und migrationsbedingte Mehrsprachigkeit, Tübingen, Narr.

KUNKEL, M. (2008), Kontrastives Arbeiten in interkulturellen Lehrertandems: « Überlegungen zum Teamteaching in Projekten doppelter Immersion », in: BURWITZ-MELZER Eva, HALLET Wolfgang, LEGUTKE Michael K., MEIßNER Franz-Joseph, Mukherjee Joybrato (éds.), Sprachen lernen Menschen bilden, Hohengehren, Schneider, pp. 165-177.

KUNKEL, M. (À. PAR.), « Reconnaissance de ressources culturelles et linguistiques allochtones. Enjeux d'un projet de double immersion en Allemagne », in : MALET Régis (éd.), Politiques de l'école et réformes curriculaires. Perspectives internationales, Bruxelles, de Boeck.

KUNKEL, M., BUDACH G. (2008), « Two-way immersion in Germany: Notes on teaching and learning practice ", in: DOOLY Melinda, EASTMENT Diana (éds.), How we're going about it. Teachers' Voices on Innovative Approaches to Teaching and Learning Languages, Newcastle upon Tyne (UK), House \& Place Cambridge Scholars Publishing, pp. 18-30.

LE PAGE, R. B., TABOURET-KELLER, A. (1985), Acts of identity. creole-based approaches to language and ethnicity, Cambridge, Cambridge University Press. 
LINDHOLM-LEARY, K. J. (2001), Dual Language Education, Clevedon et al., Multilingual Matters.

LÜDERS, C. (1995), « Von der teilnehmenden Beobachtung zur ethnographischen Beschreibung », in: König Eckard, Zedler Peter (éds.), Bilanz qualitativer Forschung. Band II: Methoden, Weinheim, Deutscher Studien Verlag, pp. 311-342.

MC'CoLLuM, P. (1999), « Learning to Value English: Cultural Capital in a Two-way Bilingual program », in: Bilingual Research Journal 23 (2/3), pp. 113-134.

NORTON, P. B. (1995), « Social identity, investment, and language learning », in: TESOL Quarterly 29 (1), pp. 9-31.

NORTON, B. (2000), Identity and language learning: Gender, ethnicity and educational change, Essex, Pearson Education Limited.

ротоWSкI, K. (2004), « Student Spanish use and investment in a dual immersion classroom:

Implications for second language acquisition and heritage language maintenance », in: Modern Language Journal, 88 (1), pp. 75-101.

ротошSк, К. (2007), Language and identity in a dual immersion school, Clevedon et al., Multilingual Matters.

ROLSTAD, K. (1997), «Effects of two way immersion on the ethnic identification of third language students. An exploratory study », in: Bilingual research journal 21 (1), pp. 43-63.

SANDFUCHS, U., ZUMHASCH C. (2002), «Wissenschaftliche Begleituntersuchung zum Schulversuch Deutsch-Italienische Grundschule Wolfsburg - Reflexionen und ausgewählte Ergebnisse », in: interkulturell 1/2, pp. 104-139.

söHN, J. (2005), Zweisprachiger Schulunterricht für Migrantenkinder. Ergebnisse der Evaluationsforschung zu seinen Auswirkungen auf Zweitspracherwerb und Schulerfolg, Berlin (http:// bibliothek.wzb.eu/pdf/2005/iv05-akibilanz2.pdf, 22.8.2009).

THOMAS WAYNE, P., COLLIER, V. (1997), School effectiveness for language minority students, Washington, DC, National Clearinghouse for Bilingual Education.

THOMAS WAYNE, P., COLLIER, V. (2002), A national study of school effectiveness for language minority students' long-term academic achievement http://crede.berkeley.edu/research/llaa/1.1_final.html, 22.8.2009

\section{NOTES}

1. Traduction française: [C]ette dévaluation de l'identité qui se réalise dans les interactions entre enseignants et élèves convainc beaucoup d'élèves de l'inefficacité de l'effort éducatif. Ils résistent à une ultérieure dévaluation de leurs identités en se retirant mentalement de la vie scolaire. [...] Pour renverser ce scénario et faire face à l'échec scolaire, il est nécessaire que les enseignants, élèves et communautés défissent ce modèle de subordination qui a caractérisé historiquement les relations dans la société, en général. Lorsque les enseignants encouragent les élèves des cultures différentes à développer la langue et la culture d'origine, à s'appuyer sur leurs expériences antérieures, ils remettent avec leurs élèves en question la representation du grand public considerant ces traits comme inférieurs ou sans valeur.

2. La conclusion que cette deuxième moitié de la classe est composée exclusivement d'enfants du groupe linguistique majoritaire sans antécédents immigrés est un malentendu: les écoles 
admettent aussi des enfants d'autres origines, sous la condition qu'ils aient des connaissances suffisantes de la langue majoritaire.

3. Cf. la banque de données du Center for Applied Linguistics (http://www.cal.org/, 15.11.2008).

4. Traduction française: [...] Je considère que la notion d'individu et celle de personnalité de l'apprenant d'une langue, dans les théories d'acquisition d'une langue seconde, doivent être reconsidérées en problématisant les distinctions dichotomiques existantes entre l'apprenant d'une langue et le contexte d'apprentissage. J'utilise le terme "identité" pour faire référence à la manière dont une personne entend sa relation avec le monde, comment cette relation est construite dans le temps et l'espace et comment la personne appréhende l'avenir. Je pense que la théorie de l'apprentissage d'une langue seconde devrait concevoir l'identité en relation aux structures sociales en général, souvent injustes et reproduites dans l'interaction sociale quotidienne.

5. Dans une méta-analyse, pourtant, Söhn 1995 met en doute la qualité de la méthodologie de quelques analyses.

6. Toutes les interviews ont été audioregistrées et transcrites entre 2005 et 2009.

7. Par contre, dans cette contribution nous n'incluons pas les extraits des interviews menées avec des élèves qui ont fait leurs propres expériences migratoires ou des élèves d'une origine autre qu'allemande ou italienne (cf. Rolstad 1997).

8. Nous avons choisi une transcription orthographique, sauf que les accentuations très fortes sont marquées par des mots complets en majuscules. Notre traduction française cherche à reproduire le style oral de l'énoncé en allemand.

9. Comme les travaux de recherche mentionnés ci-dessus l'ont démontré, les différences entre la variété standard de la langue minoritaire et les variétés parlées dans les familles des élèves peuvent mener à de graves conflits. Dans la perception de Valentina, ce n'est pas le cas. Il nous semble très important de prendre en compte les variétés dialectales des élèves d'une manière constructive, par exemple en expliquant les différences entre dialecte et standard ou en lisant et discutant la littérature dialectale, pour ne pas dévaloriser leurs connaissances transmises en salle de classe.

10. En ce sens, l'attitude de Luise semble aller au-delà des résultats de l'enquête menée par Bearse \& de Jong auxquels j'ai fait allusion ci-dessus : « Spanish is a school language for the Anglo students to be mastered for instrumental reasons; it is unclear that they adopted the values of the Latino culture in addition to learning the language and hence invested their sense of being (identity) as well. » (Bearse \& de Jong 2008, 335) Traduction française: L'espagnol, pour les élèves anglophones, est une langue de scolarisation qui doit être maîtrisée pour les raisons pratiques; il n'est pas attesté qu'en apprenant la langue, ils partagent les valeurs de la culture latine et donc investissent dans leur sens d'être (identité).

11. Tandis que Luise décrit la perspective interne, l'élève allemand Peter explique une perspective externe: « En cas de doute, les autres, la plupart des autres à l'ecole me compte parmi les italiens, parce que je suis dans cette classe. » («Im Zweifelsfall werd ich auch von den meisten dann irgendwie, von den meisten an der Schule als Italiener mit gewertet, weil ich in der Klasse bin. ») 


\section{RÉSUMÉS}

L'article traite la construction identitaire de jeunes élèves inscrits dans un projet « d'immersion réciproque " italien - allemand. Ce modèle s'adresse prioritairement aux élèves souhaitant apprendre l'italien, langue familiale, à l'école en même temps que l'allemand, langue partenaire du système éducatif. À partir d'une étude de cas, nous discuterons la construction identitaire des élèves en nous fondant sur des observations participantes en salle de classe et sur des entretiens semi narratifs. Les analyses indiquent que les élèves considèrent leur identité comme étroitement liée à leur usage linguistique, le modèle d'immersion réciproque constituant un contexte favorable pour leur investissement dans la langue et la culture italiennes. Nous montrerons que cette constatation n'est pas nécessairement en contradiction avec l'usage prédominant de l'allemand dans et en dehors de la classe.

This chapter discusses the identity construction of young pupils participating in a model of Italian-German "two-way-immersion" - a school project with a certain number of pupils sharing Italian as a family language which is also used as a "partner language " along with German in class. In this case study, we will discuss pupils' identity construction using data from participant observation in the classroom and semi-narrative interviews. The analysis shows that pupils see their identity as closely connected with the languages they use and that two-wayimmersion seems to create a beneficial context for their investment in Italian language and culture. As we will show, this observation does not necessarily contradict the prevalent use of German in and outside the classroom.

\section{INDEX}

Mots-clés : allemand, double immersion, italien, plurilinguisme, théorie subjective

\section{AUTEUR}

\section{MELANIE KUNKEL}

Universität Frankfurt am Main 